Research article

\title{
Initial human T-cell leukemia virus type 1 infection of the salivary gland epithelial cells requires a biofilm-like structure
}

Hideki Nakamura ${ }^{1, *}$, Toshimasa Shimizu ${ }^{1}$, Ayuko Takatani $^{1}$, Takashi Suematsu ${ }^{2}$, Tatsufumi Nakamura ${ }^{3}$, and Atsushi Kawakami ${ }^{1}$

${ }^{1}$ Department of Immunology and Rheumatology, Division of Advanced Preventive Medical Sciences, Nagasaki University Graduate School of Biomedical Sciences, Nagasaki, Japan

${ }^{2}$ Division of Electron Microscopy, Nagasaki University Graduate School of Biomedical Sciences, Nagasaki, Japan

${ }^{3}$ Department of Human Community, Faculty of Social Welfare, Nagasaki International University, Nagasaki, Japan

*Corresponding author: Dr. Hideki Nakamura, Department of Immunology and Rheumatology, Division of Advanced Preventive Medical Sciences, Nagasaki 
University Graduate School of Biomedical Sciences, 1-7-1 Sakamoto, Nagasaki

City, Nagasaki 852-8501, Japan. Tel.: +81-95-819-7262; Fax: +81-958-49-7270.

Email: nhideki@nagasaki-u.ac.jp

Short title: Initial HTLV-1 infection toward SGECs

Key words: HTLV-1, salivary gland epithelial cell, biofilm, T cell 


\section{SUMMARY}

The initial phase of the human T cell leukemia virus-1 (HTLV-1) infection of salivary gland epithelial cells (SGECs) was examined. SGECs of patients with Sjögren's syndrome (SS) and non-SS subjects were co-cultured with the HTLV-1-infected cell line HCT-5 or MOLT-4, then immunofluorescence (IF), scanning and transmission electron microscopy (SEM/TEM) were employed.

The extracellular matrix and linker proteins galectin-3, agrin, and tetherin were expressed on the surfaces of both HCT-5 and MOLT-4 cells. HTLV-1 Gag-positive spots were observed on adjacent SGECs after $1 \mathrm{~h}$ of co-culture with HCT-5. Both in subjects with and those without SS, agrin and tetherin were co-expressed with HTLV-1 Gag on SGECs after co-culture with HCT-5, although no polarization of HTLV-1 Gag and relevant molecules was observed. SEM showed HTLV-1 virions that were found on HCT-5 were observed in the interfaces between HCT-5 cells and SGECs. TEM imaging showed that HTLV-1 virions were transmitted to SGECs at the interface with thin film-like structure, while HTLV-1 virions were released from the surface of HCT-5 cells. No endogenous retroviruses were observed. These results showed that the initial phase of HTLV-1 infection toward SGECs of SS was mediated not by viral 
synapses, but by biofilm-like components.

(196 words) 
Human T-cell leukemia virus-1 (HTLV-1) is a retrovirus that is directly involved in the etiology of adult T-cell leukemia (ATL), HTLV-1-associated myelopathy (HAM), and uveitis $(1,2)$. Regarding the relationship between autoimmune disease and HTLV-1, we demonstrated that tumor necrosis factor-alpha (TNF- $\alpha$ ) inhibitors showed diminished efficacy for patients with HTLV-1-seropositive rheumatoid arthritis (3). Sjögren's syndrome (SS) is an autoimmune disease characterized by sicca symptoms, and the presence of anti-Ro/SS-A and anti-La/SS-B antibodies $(4,5)$, has been reported to be associated with some viruses, including Epstein-Barr virus (6) and cytomegalovirus (7). However, SS has not been recognized as part of the group of HTLV-1-associated diseases.

Epidemiologically, a relationship between HTLV-1 and SS has been reported. In Nagasaki, an endemic area of HTLV-1, a high prevalence of HTLV-1 in SS patients (23\%) was reported among blood donors $(8,9)$. In studies in 1997 and 2015, we reported a high prevalence of SS in our patients with HAM irrespective of the classification criteria applied $(10,11)$. In another study, we sought direct evidence of HTLV-1 infection in SS, and found that the salivary gland epithelial cells (SGECs) of patients with SS were infected with HTLV-1 (12). Co-culture of HTLV-1-infected cells and SGECs for 72-96 h revealed 
positive expressions of the HTLV-1 Gag proteins including $\mathrm{p} 19, \mathrm{p} 28$ that is one of gag protein intermediates, and Gag (HTLV-1 Gag) and elevated expressions of cytokines and chemokines such as soluble intercellular adhesion molecule 1 (ICAM-1), regulated on activation, normal $\mathrm{T}$ cell expressed and secreted (RANTES), and interferon $\gamma$-induced protein 10-kd (IP-10) in both the culture medium and SGECs. However, the initial phase of the HTLV-1 infection of SGECs remained unclear.

With respect to the HTLV-1 infection of CD4+ T lymphocytes, there are two major theories. Regarding the cell-to-cell infection of HTLV-1, the concept of virological synapses (VSs) was posited in a study by Igakura et al. (13), in which HTLV-1 viral proteins and genomes were reported to accumulate at the interface between HTLV-1-infected cells and target lymphocytes, accompanied by the polarization of ICAM-1 and lymphocyte function-associated antigen (LFA-1). The other theory involves the biofilm-like aggregation of extracellular matrix and cellular linker proteins, with the concept that aggregation of these proteins assist in the viral transmission (14). We conducted the present study to determine whether or not VSs or biofilm-like structures are directly associated with the transmission of HTLV-1 virions to SGECs obtained from patients with 
SS as well as to SGECs from non-SS subjects.

\section{MATERIAL AND METHODS}

\section{Patients with Sjögren's syndrome}

SGECs from labial salivary glands (LSGs) were obtained from twelve patients with suspected SS (all female; age: $58.8 \pm 11.5$ years). The classification of SS was performed based on the revised criteria proposed by the American-European Consensus Group (AECG) (15). All eight patients (age: 58.6 \pm 11.4 years) with pSS based on the AECG criteria were HTLV-1-seronegative as determined by a chemiluminescent enzyme immunoassay (CLEIA). Four non-SS subjects were defined as cases that did not fulfill the above classification criteria although they had xerostomia or xerophthalmia.

\section{Antibodies and reagents}

Rabbit anti-agrin antibody and goat anti-BST-2 (also known as tetherin) antibody were purchased from Santa Cruz Biotechnology (Santa Cruz, CA). Mouse anti-HTLV-1 (p19, p28, and Gag) antibodies were purchased from Chemicon International (Temecula, CA). Rabbit anti-galaectin-3 antibody, anti-talin 
antibody, rabbit anti-alpha tubulin antibody, and mouse ICAM-1 antibody were purchased from Proteintech (Rosemont, IL). Rabbit anti-LFA-1 (also known as ITGB2) antibody was purchased from Lifespan BioSciences (Seattle, WA). Secondary antibodies including donkey anti-mouse $\operatorname{IgG}$ conjugated with fluorescein isothiocyanate (FITC) and donkey anti-rabbit IgG conjugated with tetramethyl rhodamine isothiocyanate (TRITC) were purchased from Jackson ImmunoResearch Laboratories (West Grove, PA). Hoechst dye 33258 was purchased from Sigma (St. Louis, MO). Mouse anti-tetherin (CD317, PDCA-1) neutralizing antibody was purchased from Thermo Fisher Scientific (Waltham, MA). Monoclonal rabbit anti-human cytokeratin 8/18 antibody mouse $\operatorname{IgG1}$, normal rabbit serum and normal goat serum were purchased from Dako Cytomation (Glostrup, Denmark).

\section{Salivary gland biopsy and cell culture of epithelial cells}

A biopsy for the LSGs of the enrolled subjects was performed at the lower lip under local anesthesia. Some LSG samples were sent for the pathological diagnosis of sialadenitis. As reported previously (12), the remaining samples were used for the culture of SGECs in a defined keratinocyte-serum-free 
medium (SFM) culture medium (Invitrogen Life Technologies, Carlsbad, CA) supplemented with hydrocortisone (Sigma) and bovine pituitary extract (Kurabo, Osaka, Japan).

For the 1-hr co-cultures with SGECs in the defined keratinocyte-SFM culture medium, we used the HTLV-1-infected T-cell line HCT-5 (16, 17), which is derived from cerebrospinal fluid cells of a patient with HAM, and the non-HTLV-1 infected T-cell line MOLT-4. HCT-5 cells were subcultured in 20\% fetal bovine serum (FBS) in RPMI 1640 medium supplemented with interleukin (IL)-2. MOLT-4 cells were maintained in RPMI 1640 medium with 10\% FBS. Before the co-culturing, HCT-5 and MOLT-4 cells were cultured in 100-mm dishes. Our use of human samples following informed consent from the subjects was conducted with the approval of the Ethics Committee of Nagasaki University Hospital (approval no. 09102822-4).

\section{Immunofluorescence}

An immunofluorescence was performed as described previously (18). Briefly, SGECs were cultured on type I collagen-coated cover slips. After the co-culture with $1 \times 10^{6}$ cells of the HCT-5 or MOLT-4 cells, SGECs were washed with 
phosphate-buffered saline (PBS) three times and fixed in phosphate-buffered saline (PBS) containing 4\% paraformaldehyde and immersed in methanol. After blocking in 5\% normal horse serum in PBS, the SGECs were incubated with the primary antibodies for $1 \mathrm{~h}$ at room temperature. Mouse IgG1, normal rabbit serum, and normal goat serum were used as isotype-matched controls.

The cells were then incubated with FITC-conjugated and TRITC-conjugated secondary antibodies under dark conditions. The images were scanned using a fluorescence microscope (BZ-X710; Keyence, Osaka, Japan). The immunostaining of HCT-5 and MOLT-4 cells was performed in the same manner as described above for SGECs. For the optical microscopy observation of HCT-5 and MOLT-4 cells, an Olympus CK40 microscope was used. For improvement of images captured by BZ-X710, we analyzed by using haze reduction function that was introduced in previous reports $(19,20)$.

\section{Scanning electron microscopy (SEM)}

For the scanning electron microscopy examination, $1 \times 10^{6}$ HCT- 5 or MOLT- 4 cells co-cultured with SGECs that were seeded on cover slips were washed with 0.1 M phosphate buffer at room temperature five times for 10 min each time and 
fixed in $2.5 \%$ glutaraldehyde in $0.1 \mathrm{M}$ phosphate buffer. After being washed with $0.1 \mathrm{M}$ phosphate buffer four times, the samples were post-fixed for $2 \mathrm{~h}$ in $1 \%$ osmium tetroxide in $0.1 \mathrm{M}$ phosphate buffer, then washed twice with $0.1 \mathrm{M}$ phosphate buffer and dehydrated through a series of $50 \%, 70 \%$ and $80 \%$ ethanol solutions for $10 \mathrm{~min}$ at $4{ }^{\circ} \mathrm{C}$, followed by $90 \%, 95 \%$ and $99.5 \%$ ethanol solutions at room temperature. After substitution displacement with t-butyl alcohol at $40^{\circ} \mathrm{C}$ for $20 \mathrm{~min}$ each time, the samples were dried with vacuum freeze-drying equipment. Platinum film was used to cover the dried samples by sputter-coating (JFC-1600JEOL). The samples were mounted on a specimen support stage with carbon tape. A secondary electron image (SEI) or a lower secondary electron image (LEI) was captured by a JEOL JSM-6700F microscope with an emission gun operating at $8 \mathrm{kV}$. Regarding immune electron microscopy for SEM, HCT-5 pellet was fixed in 4\% paraformaldehyde (PFA) and dropped on carbon coating slides. After blocking with $1 \%$ bovine serum albumin in PBS, samples were incubated with or without mouse anti-HTLV-1 Gag antibody followed by incubation with anti-mouse secondary antibody conjugated with $10 \mathrm{~nm}$ gold particle. 


\section{Transmission electron microscopy (TEM)}

First, $1 \times 10^{6}$ HCT- 5 cells co-cultured with SGECs that were seeded on a collagen-coated chamber slide (Matsunami, Osaka, Japan) were washed with 0.1 M phosphate buffer at room temperature five times and fixed hermitically in $2.5 \%$ glutaraldehyde in $0.1 \mathrm{M}$ phosphate buffer at $4{ }^{\circ} \mathrm{C}$ for $4 \mathrm{~h}$. After being washed with $0.1 \mathrm{M}$ phosphate buffer four times, the samples were post-fixed for $2 \mathrm{~h}$ in $1 \%$ osmium tetroxide in $0.1 \mathrm{M}$ phosphate buffer and then washed twice with $0.1 \mathrm{M}$ phosphate buffer and dehydrated through a series of $50 \%, 70 \%$ ethanol solutions for 10 min at $4{ }^{\circ} \mathrm{C}$, followed by $80 \%, 90 \%, 95 \%, 99.5 \%$ and $100 \%$ ethanol solutions at room temperature. We created an epoxide resin that consisted of a base compound, curative agent and accelerating agent, and then filled a beam capsule with the epoxide resin. The beam capsule was inverted on the chamber slides, and the samples were thermal polymerized at $50^{\circ} \mathrm{C}$ for $72 \mathrm{~h}$. Each detached specimen with polymerized epoxy resin was sliced thinly using an ultramicrotome (ULTRACUT Leica, Wetzlar, Germany). Counterstaining of the sections was performed by using uranyl acetate and lead nitrate. Images were captured by a JEOL JEM-1200EX microscope for the TEM examination. 
Regarding immune electron microscopy for TEM, HCT-5 pellet was fixed in 4\% paraformaldehyde (PFA) and dehydrated. Then, heat polymerization was performed in LR white resin (London Resin Company Ltd., UK) for post embedding staining.

\section{RESULTS}

Co-expression of extracellular matrix and linker proteins on SGECs after co-culture

Extracellular matrix proteins including galectin-3, agrin and tetherin were expressed on the surface of HCT-5 cells (Fig. 1A). These three molecules were co-expressed with HTLV-1 Gag, although the co-expression was limited to a small area. Although MOLT-4 cells also expressed galectin-3, agrin, and tetherin (Fig. 1B), the expression of these molecules was weaker compared to that on HCT-5 cells. No expression of HTLV-1 Gag on MOLT-4 cells was observed. The HCT-5 cells showed some emitted dots around the cells (Fig. 2A). After the 1-hr co-culture of SGECs and HCT-5 cells, dot signals of HTLV-1 Gag were observed 
on the surface of cytokeratin 8/18-positive SGECs adjacent to HCT-5 cells (Fig. 2B), although these Gag signals were not observed in the nuclei of the SGECs. In contrast, the SGECs co-cultured with MOLT-4 cells showed no dotted staining of HTLV-1 Gag proteins (Fig. 2C). In addition, only the MOLT-4 cells lacked HTLV-1 Gag protein expression (Fig. 2C). Without co-culturing, no expression of Gag protein was observed on SGECs (Suppl. Fig. S1A). After the 1-hr co-culture of SGECs with HCT-5 cells, no co-expression of galectin-3 and HTLV-1 Gag signal was observed on SGECs, although galectin-3 was expressed on SGECs (Fig. 3A).

In contrast, agrin and tetherin were co-expressed with HTLV-1 Gag, and tetherin in particular showed a high frequency of co-expression compared to the co-expressing area with agrin (Fig. 3A). The co-expression of Gag protein and galectin-3, agrin, and tetherin on SGECs in other cases is shown in Supplementary Figure S1B, in which the co-expression of Gag protein with agrin and tetherin was observed as mentioned above. The expressions of galectin-3, agrin, and tetherin on SGECs before co-culturing with HCT-5 cells were not significantly different from those after the co-culture (Suppl. Fig. S1A). When SGECs were co-cultured with MOLT-4 cells, no HTLV-1 Gag signal was 
observed, although galectin-3 and agrin were expressed on the MOLT-4 cells (Fig. 3B). These findings were similar whether the SGECs were derived from patients with pSS or non-SS subjects. To confirm importance of biofilm-like structure, we showed inhibition test toward tetherin. By using $2 \mu \mathrm{g} / \mathrm{ml}$ and $20 \mu \mathrm{g} / \mathrm{ml}$ neutralizing antibody toward tetherin, no obvious reduced expression of tetherin on both HCT-5 and SGECs was observed after co-culture with HCT-5 and SGECs at 1 and $24 \mathrm{~h}$ (Suppl. Fig. S2).

No virological synapse formation in the co-cultures of SGECs with HCT-5 cells After the 1-hr co-culture of SGECs with HCT-5, no polarization of HTLV-1 Gag proteins was observed at the contact surface of these cells (Fig. 4A). In addition, no accumulation of talin (one of the structural components of VSs) was observed at the interface. No polarization of another component, $\alpha$-tubulin, was observed after co-culture with HCT-5 cells (Fig. 4A). The expression of ICAM-1 was observed on the surface of HCT-5 cells, but there was no polarization of ICAM-1 on HCT-5 cells and LFA-1 on SGECs at the interface. The SGECs co-cultured 
with MOLT-4 cells showed no polarization of talin and LFA-1 without the expression of HTLV-1 Gag and ICAM-1 signals (Fig. 4B). These findings were similar whether the SGECs were derived from patients with pSS or non-SS subjects.

We observed the expression of VS-related proteins as well as extracellular matrix and linker proteins before the co-culturing (Suppl. Fig. S1).

\section{Detection of HTLV-I virions on the surface of co-cultured SGECs}

Although HCT-5 cells have a more cilia-like structure on the surface (Fig. 5A) under an optical microscope compared to MOLT-4 cells (Fig. 5B), the SEM images showed a complicated surface (Fig. 5C) with many particles of approximately 100-nm diameter with different sizes (Fig. 5D). Immune electron microscopy for HCT-5 pellet showed that compared to control staining without primary antibody (Fig. 5E) some gold particles were detected in backscattered electron image (BEI) by incubation with anti- HTLV-1 Gag antibody followed by 10nm gold particle conjugated secondary antibody (Fig. 5F). HCT-5 cells with a number of foot processes contacted SGECs (Fig. 5EG,FH). SEM images also 
showed that small particles (approximately $100 \mathrm{~nm}$ in diameter) were present in the area between HCT-5 cells and SGECs (Fig. 5GI,HJ).

TEM images revealed HTLV-1 virions with an envelope on the surface of HCT-5 cells (Fig. 6A). Differences in the sizes of the virions were observed, and the budding of hemispherical virions was seen on the surface membrane of HCT-5 cells, showing one of the characteristics of a type $\mathrm{C}$ retrovirus (Fig. 6B). Immune electron microscopy for HCT-5 pellet showed that compared to control staining without primary antibody (Fig. 6C) many gold particles were detected by incubation with mouse anti-HTLV-1 Gag antibody followed by $10 \mathrm{~nm}$ gold particle conjugated secondary antibody (Fig. 6D). The TEM imaging showed foot processes on the surface at the junction of HCT-5 cells and SGECs (Fig. 6EE), and the release of HTLV-1 virions occurred among other cellular components (Fig. 6PF). At high magnification, virions of approximately 100-nm diameter were detected on the HCT-5 side (Fig. 6EG). Similarly, virions were also detected at the SGEC side (Fig. 6FH). In addition, a thin film-like structure was observed on the surface of HCT-5 cells (Fig. 6A right panel, 6PF) and SGECs (Fig. 6FH).

We also confirmed that there were no released virions at the interface between 
MOLT-4 cells and SGECs by SEM and TEM in a control experiment (Suppl. Fig.

S3). Although typical SGECs had microvilli on their surface (Suppl. Fig. S3B,F), the MOLT-4 cells had an irregular surface and remarkable notch on the nucleus (Suppl. Fig. S3A,E) and made smooth contacts with the SGECs (Suppl. Fig. S3C,D,G,H). We also confirmed that there were no endogenous retroviruses on the surface of the SGECs (Suppl. Fig. S3B).

\section{DISCUSSION}

Our findings revealed that, unlike VSs, the biofilm-like structure of extracellular and linker proteins facilitated the transmission of HTLV-1 virions to SGECs. With respect to definition of initial phase regarding contact between HTLV-1-infected cell line and non-immune SGECs, there was no clear standard. Therefore, we drew upon the descriptions by Pais-Correia (14), in which important molecules or time course were demonstrated for experiments between HTLV-1-infected and uninfected lymphocytes. With respect to autoimmune diseases, including SS and rheumatoid arthritis, some reports have described a relationship between HTLV-1 infection and these disorders $(21,22)$. With regard to the direct infection of non-lymphocytes by HTLV-1, some in vitro studies have 
used retinal glial cells or synovial cells $(23,24)$, but their analyses were of $3-$ 7-day co-cultures with an HTLV-1-infected cell line. In our study of SGECs co-cultured with HCT-5 cells, the cytoplasmic expression of HTLV-1 Gag proteins was observed at $\geq 72 \mathrm{~h}(12)$. The expression of HTLV-1 Gag proteins in these studies was thought to occur in response to the integration of HTLV-1 into the host cell genome. Our findings demonstrating that galectin-3 showed no co-expression with HTLV-1 Gag appear to differ from the previous reported on HTLV-1-infected lymphocytes, in which galectin-3 and HTLV-1 Gag were co-expressed on the surface of HTLV-1-infected lymphocytes (14). However, our findings suggest the possibility that at least two molecules are involved in the assembly of extracellular matrix proteins with HTLV-1 virions. Although neutralizing test with anti-tetherin antibody showed no obvious effect, functional activity of tetherin was not determined. Regarding viral biofilms involved in HTLV-1 infection, Tarasevich et al. (25) reported the up-regulation of CD4, CD150, CD25, and CD80 in biofilms in response to HTLV-1 infection. Regarding the expression of the tax gene in HCT-5 cells, we observed a detectable basal-level expression of both tax as well as HTLV-1 bZIP factor (HBZ), and proviral load before the addition of TNF inhibitors (26), indicating 
that tax might be associated with the expression of cytokines in the HCT-5 cell line.

Although HTLV-1 was mainly transmitted between HTLV-1-infected cells and uninfected lymphocytes, other types of cells should be examined in this context, including dendritic cells (DCs) and other epithelial cells. The involvement of DCs should be considered because cell-free HTLV-1 virions have been shown to have the potential to infect DCs (27). Since a cell-free HTLV-1 infection of plasmatoid DCs (pDCs) was reported to transfer HTLV-1 virions to CD4+ T cells (28), the role of pDCs in SS might be crucial for the effective in vivo transmission of HTLV-1 virions between HTLV-1-infected and HTLV-1-uninfected cell types. Regarding cell-free HTLV-1 infection and transmission toward DCs, the involvement of a DC-specific intracellular adhesion molecule-3-grabbing nonintegrin (DC-SIGN) was reported (29), suggesting that a DC-mediated viral transmission system might be involved in cases of HTLV-1 infection of salivary gland epithelial cells. Recent study with respect to relationship between innate immunity and HTLV-1 infection also showed importance of HTLV-1-related glycan-rich viral structure that is mediated by antiviral response detected in pDCs (30). From a view point of 
relationship between saliva and HTLV-1 infection, simian T-leukemia virus type 1 (STLV-1) and simian foamy virus (SFV) in saliva of primates except humans are shown (31). Because STLV-1 and SFV in saliva of primates was reported to transmit to blood of recipients as humans, role of saliva might have a crucial role for transmission of retroviruses.

With regard to VSs, it was reported (13) that the polarization of a microtubule organizing center (MTOC) $(32,33)$ and the accumulation of talin (one of the cytoskeletal proteins) played crucial roles in the delivery of HTLV-1 virions from HTLV-1-infected cells to uninfected cells. Human immunodeficiency virus type -1 (HIV-1) also uses VSs with the polarization of virus proteins (34), and two components, the Gag capsid protein and envelope glycoproteins, were shown to assist in the turnover of HIV-1 VSs. It was also reported that in a VS model in HIV-1, LFA-1 had the potential to polarize VS and recruit an MTOC (35). From the expression of LFA-1 on SGECs, we should take cellular conduits into consideration. It was reported (36) that HTLV-1-encoded $\mathrm{p} 8$ protein assisted HTLV-1 transmission toward uninfected T lymphocytes. They showed HTLV-1 was transmitted by cellular conduits that were filopodium-like protrusion with $\mathrm{p} 8$ that was cleaved from precursor p12. Because foot processes were observed on 
the surface of HCT-5 at the interface, the presence of cellular conduits should be considered. Because they demonstrated clustering of LFA-1 was required for cellular conduits formation, the findings were different from our observations that indicated no accumulation of LFA-1 at the interface in $1 \mathrm{~h}$ co-culture. However, we should carefully consider the possibility for the presence of cellular conduits because they observed clustering of LFA-1 at 20 minutes co-culture. We observed no cellular conduits formation at 0 and 3 minutes, but short cellular conduits appeared at 20 minutes without accumulation of LFA-1 (Suppl. Fig. S4). Finally, we found longer cellular conduits formation toward adjacent SGEC at 60 minutes. Although the defective accumulation of LFA-1 or time points was different from the previous report (36), similar condition might exist between HCT-5 and SGECs.

With respect to the HTLV-1 infection of epithelial cells, a previous report demonstrated the HTLV-1 infection of enterocytes with microvilli using TEM (37), and also showed that free virions were present at the interface between HTLV-1-infected cells and epithelial cells. In follicular epithelial cells from HTLV-1-positive individuals with Hashimito's thyroiditis, no HTLV-1 particles were detected by electron microscopy despite the detection of HTLV-1 viral 
proteins and mRNA (38). That finding differs from the results of our previous study (39), which demonstrated the expression of HBZ in salivary epithelial cells by in situ hybridization. Therefore, it might be difficult to detect HTLV-1-associated molecules or virions in different epithelial cell types or under different specimen-processing conditions.

With regard to the detection of retrovirus by TEM, Yamano et al. (40) reported that they observed A-type-like retroviral particles in SGECs of SS patients with positive reverse transcriptase activity. However, the current understanding is that HTLV-1 infection is not confirmed unless the SS patient is positive for anti-HTLV-1 antibody. We have also considered the possibility of human endogenous retroviruses (HERVs) because HERVs were detected in a teratocarcinoma cell line $(41,42)$. Since we detected no virus-like particles on the SGECs that were co-cultured with MOLT-4 cells despite extensive observations, we confirmed that the type C virions on SGECs that were co-cultured with HCT-5 cells were not HERVs.

This study has some limitations. The routes over which HTLV-1 virions travelled from the surface of SGECs to the nucleus were not elucidated. 
In addition, due to technical problems during the preparation process, we did not label HTLV-1 proteins toward samples with SGECs with immunogold particles for the electron microscopy. However, we could confirm that the particles on HCT-5 only samples were HTLV-1 by using anti-mouse secondary antibody conjugated with gold particles. With regard to neutralizing test, affinity of neutralizing antibodies toward tetherin antigen might be associated with negative results in Suppl. Fig S2. In a future study, we plan to investigate the possibility of the interaction of other immune cells such as or macrophages in vivo or the aforementioned dendritic cells.

Taken together, our present findings provide the first description of the initial dynamic state of HTLV-1 virions at the interface between HCT-5 cells and SGECs. We elucidated that part of the biofilm-like assemblies of the extracellular matrix and linker proteins played a crucial role in transmitting the HTLV-1 released by HTLV-1-infected cells to the SGECs.

(Word count: 36592978)

\section{Key messages}

The HTLV-1-infected cells have the extracellular matrix and linker proteins on 
their surface.

Biofilm-like components including agrin and tetherin were involved in transmission of HTLV-1 toward SGECs.

An electron microscopy confirmed transmission of virions at the interface between HCT-5 cells and SGECs.

\section{Conflicts of interest:}

All authors declare no conflicts of interest in this paper.

\section{Acknowledgements:}

We thank Ms. Kaori Furukawa for her technical assistance. This work was supported in part by a Grant from The Bristol-Myers Squibb Company.

\section{Author contributions:}

All authors were involved in drafting the article or revising it critically for important intellectual content, and all authors approved the final version for 
publication. Dr. Hideki Nakamura has full access to all of the data in the study and takes responsibility for the integrity of the data and the accuracy of the data analysis.

Study conception and design: $H$. Nakamura

Salivary gland biopsy: H. Nakamura, T. Shimizu, A. Takatani Acquisition of data: T. Suematsu, H. Nakamura

Analysis and interpretation of data: H. Nakamura, T. Nakamura, A.

Kawakami 


\section{References}

1. Yasunaga J, and Matsuoka M. Molecular mechanisms of HTLV-1 infection and pathogenesis. Int J Hematol 2011;94:435-42

2. Terada Y, Kamoi K, Komizo T, Miyata K, Mochizuki M. Human T cell leukemia virus type 1 and eye diseases. J Ocul Pharmacol Ther 2017;33:21623.

3. Suzuki T, Fukui S, Umekita K, Miyamoto J, Umeda M, Nishino A, et al. Attenuated effectiveness of tumor necrosis factor inhibitors for anti-human $\mathrm{T}$ lymphotropic virus type I antibody-positive rheumatoid arthritis. Arthritis Rheumatol 2018;70:1014-21.

4. Mariette X, and Criswell LA. Primary Sjögren's Syndrome. N Engl J Med 2018; 378:931-9.

5. Nakamura H., Kawakami A., Eguchi K. Mechanisms of autoantibody production and the relationship between autoantibodies and the clinical manifestations in Sjögren's syndrome. Transl Res 2018;148:281-8.

6. Inoue H, Mishima K, Yamamoto-Yoshida S, Ushikoshi-Nakayama R, Nakagawa Y, Yamamoto K, et al. Aryl hydrocarbon receptor-mediated 
induction of EBV reactivation as a risk factor for Sjögren's syndrome. J Immunol 2012;188:4654-62.

7. Ohyama Y, Carroll VA, Deshmukh U, Gaskin F, Brown MG, Fu SM. Severe focal sialadenitis and dacryoadenitis in NZM2328 mice induced by MCMV: A novel model for human Sjögren's syndrome. J Immunol 2006;177:7391-7.

8. Terada K, Katamine S, Eguchi K, Moriuchi R, Kita M, Shimada H, et al. Prevalence of serum and salivary antibodies to HTLV-1 in Sjögren's syndrome. Lancet 1994;344:1116-9.

9. Hida A, Imaizumi M, Sera N, Akahoshi M, Soda M, Maeda R, et al. Association of human T lymphotropic virus type I with Sjogren syndrome. Ann Rheum Dis 2010;69:2056-7.

10. Nakamura H, Eguchi K, Nakamura T, Mizokami A, Shirabe S, Kawakami A, et al. High prevalence of Sjögren's syndrome in patients with HTLV-I associated myelopathy. Ann Rheum Dis 1997;56:167-72.

11 Nakamura H, Shimizu T, Takagi Y, Takahashi Y, Horai Y, Nakashima Y, et al. Reevaluation for clinical manifestations of HTLV-I-seropositive patients with Sjögren's syndrome. BMC Musculoskelet Disord 2015;16:335. 
12. Nakamura H, Takahashi Y, Yamamoto-Fukuda T, Horai Y, Nakashima Y, Arima K, et al. Direct infection of primary salivary gland epithelial cells by human T lymphotropic virus type I in patients with Sjögren's syndrome. Arthritis Rheumatol 2015;67:1096-106.

13. Igakura T, Stinchcombe JC, Goon PK, Taylor GP, Weber JN, Griffiths GM, et al. Spread of HTLV-I between lymphocytes by virus-induced polarization of the cytoskeleton. Science 2003; 299:1713-6.

14. Pais-Correia AM, Sachse M, Guadagnini S, Robbiati V, Lasserre R, Gessain A, et al. Biofilm-like extracellular viral assemblies mediate HTLV-1 cell-to-cell transmission at virological synapses. Nat Med 2010; 16:83-9.

15. Vitali C, Bombardieri S, Jonsson R, Moutsopoulos HM, Alexander EL, Carsons SE, et al.; European Study Group on Classification Criteria for Sjögren's Syndrome. Classification criteria for Sjögren's syndrome: A revised version of the European criteria proposed by the American-European Consensus Group. Ann Rheum Dis 2002;61:554-8.

16. Nakamura T, Tsujihata M, Shirabe S, Matsuo H, Ueki Y, Nagataki S. Characterization of HTLV-I in a T-cell line established from a patient with myelopathy. Arch Neurol 1989; 46:35-7. 
17. Fukushima N, Nakamura T, Nishiura Y, Ida H, Aramaki T, Eguchi K. HTLV-I production based on activation of integrin/ligand signaling in HTLV-I-infected T cell lines derived from HAM/TSP patients. Intervirology $2008 ; 51: 123-9$.

18. Nakamura H, Kawakami A, Iwamoto N, Ida H, Koji T, Eguchi K. Rapid and significant induction of TRAIL-mediated type II cells in apoptosis of primary salivary epithelial cells in primary Sjögren's syndrome. Apoptosis 2008; 13:1322-30.

19. Yamasaki S, Ishikawa E, Sakuma M, Ogata K, Sakata-Sogawa K, Hiroshima $\mathrm{M}$ et al. Mechanistic basis of pre-T cell receptor-mediated autonomous signaling critical for thymocyte development. Nat Immunol. 2006;7:67-75.

20. Okada Y, Ueshin Y, Isotani A, Saito-Fujita T, Nakashima H, Kimura K et al. Complementation of placental defects and embryonic lethality by trophoblast-specific lentiviral gene transfer. Nat Biotechnol. 2007;25:233-7.

21. Umekita K, Hidaka T, Miyauchi S, Ueno S, Kubo K, Takajo I, et al. Treatment with anti-tumor necrosis factor biologic agents in human $\mathrm{T}$ lymphotropic virus type I-positive patients with rheumatoid arthritis. Arthritis Care Res (Hoboken) 2014; 66:788-92. 
22. Vale DAD, Casseb J, de Oliveira ACP, Bussoloti Filho I, de Sousa SCOM, Ortega KL. Prevalence of Sjögren's syndrome in Brazilian patients infected with human T-cell lymphotropic virus. J Oral Pathol Med 2017; 46:543-8.

23. Sato Y, Ito K, Moritoyo T, Fujino Y, Masuda K, Yamaguchi K, et al. Human T-cell lymphotropic virus type 1 can infect primary rat retinal glial cells and induce gene expression of inflammatory cytokines. Curr Eye Res 1997; 116:782-91.

24. Sakai M, Eguchi K, Terada K, Nakashima M, Yamashita I, Ida H, et al. Infection of human synovial cells by human $\mathrm{T}$ cell lymphotropic virus type I. Proliferation and granulocyte/macrophage colony-stimulating factor production by synovial cells. J Clin Invest 1993; 92:1957-66.

25. Tarasevich A, Filatov A, Pichugin A, Mazurov D. Monoclonal antibody profiling of cell surface proteins associated with the viral biofilms on HTLV-1 transformed cells. Acta Virol 2015; 59:247-56.

26. Fukui S, Nakamura H, Takahashi Y, Iwamoto N, Hasegawa H, Yanagihara K, et al. Tumor necrosis factor alpha inhibitors have no effect on a human T-lymphotropic virus type-I (HTLV-I)-infected cell line from patients with HTLV-I-associated myelopathy. BMC Immunol 2017; 18:7. 
27. Alais S, Mahieux R, Dutartre H. Viral source-independent high susceptibility of dendritic cells to human T-cell leukemia virus type 1 infection compared to that of T lymphocytes. J Virol 2015; 89:10580-90.

28. Jones KS, Petrow-Sadowski C, Huang YK, Bertolette DC, Ruscetti FW Cell-free HTLV-1 infects dendritic cells leading to transmission and transformation of CD4(+) T cells. Nat Med 2008;14:429-36.

29. Jain P, Manuel SL, Khan ZK, Ahuja J, Quann K, Wigdahl B. DC-SIGN mediates cell-free infection and transmission of human T-cell lymphotropic virus type 1 by dendritic cells. J Virol 2009; 83:10908-21.

30. Assil S, Futsch N, Décembre E, Alais S, Gessain A, Cosset FL et al. Sensing of cell-associated HTLV by plasmacytoid dendritic cells is regulated by dense $\beta$-galactoside glycosylation. PLoS Pathog. 2019;15:e1007589.

31. Alais S, Pasquier A, Jegado B, Journo C, Rua R, Gessain A et al. STLV-1 co-infection is correlated with an increased SFV proviral load in the peripheral blood of SFV/STLV-1 naturally infected non-human primates. PLoS Negl Trop Dis. 2018;12:e0006812.

32. Nejmeddine M, Barnard AL, Tanaka Y, Taylor GP, Bangham CR. 
Human T-lymphotropic virus, type 1, tax protein triggers microtubule reorientation in the virological synapse. J Biol Chem 2005; 280:29653-60.

33. Nejmeddine M, Negi VS, Mukherjee S, Tanaka Y, Orth K, Taylor GP, et al. HTLV-1-Tax and ICAM-1 act on T-cell signal pathways to polarize the microtubule-organizing center at the virological synapse. Blood 2009; $114: 1016-25$.

34. Gardiner JC, Mauer EJ, Sherer NM. HIV-1 Gag, envelope, and extracellular determinants cooperate to regulate the stability and turnover of virological synapses. J Virol 2016; 90:6583-97.

35. Starling S, and Jolly C. LFA-1 Engagement triggers T cell polarization at the HIV-1 virological synapse. J Virol 2016; 90:9841-54.

36. Van Prooyen N, Gold H, Andresen V, Schwartz O, Jones K, Ruscetti F et al. Human T-cell leukemia virus type $1 \mathrm{p} 8$ protein increases cellular conduits and virus transmission. Proc Natl Acad Sci U S A. 2010;107:20738-43.

37. Zacharopoulos VR, Perotti ME, Phillips DM. Lymphocyte-facilitated infection of epithelia by human T-cell lymphotropic virus type I. J Virol 1992; 66:4601-5. 
38. Kawai H, Mitsui T, Yokoi K, Akaike M, Hirose K, Hizawa K, et al. Evidence of HTLV-I in thyroid tissue in an HTLV-I carrier with Hashimoto's thyroiditis. J Mol Med (Berl) 1996; 74:275-8.

39. Nakamura H, Hasegawa H, Sasaki D, Takatani A, Shimizu T, Kurushima S, et al. Detection of human T lymphotropic virus type-I bZIP factor and tax in the salivary glands of Sjögren's syndrome patients. Clin Exp Rheumatol $2018 ; 112: 51-60$.

40. Yamano S, Renard JN, Mizuno F, Narita Y, Uchida Y. Retrovirus in salivary glands from patients with Sjögren's syndrome. J Clin Pathol 1997;50:223-30.

41. Löwer R, Boller K, Hasenmaier B, Korbmacher C, Müller-Lantzsch N, Löwer J, et al. Identification of human endogenous retroviruses with complex mRNA expression and particle formation. Proc Natl Acad Sci USA 1993; 90:4480-4.

42. Grandi N, Tramontano E. Human endogenous retroviruses are ancient acquired elements still shaping innate immune responses. Front Immunol 2018; 10;9:2039. 


\section{Figure legends}

Fig. 1. Expression of biofilm component in HCT-5 cells. A: Expression of extracellular matrix and linker proteins on HCT-5 cells. After fixation, the SGECs were reacted with primary antibodies including mouse anti-HTLV-1 Gag antibody (mGag), rabbit anti-galectin-3 (rgalectin-3), agrin antibodies (ragrin) and goat anti-tetherin antibody (gtetherin) followed by FITC-conjugated anti-mouse and TRITC-conjugated anti-rabbit or goat secondary antibodies. Arrowheads: Extracellular matrix and linker proteins, Gag and co-expression on the surface of HCT-5 cells. B: Expression of extracellular matrix and linker proteins on MOLT-4 cells. The same primary and secondary antibodies were used as for HCT-5 cells. Bright field images were simultaneously captured. Representative results of five independent experiments with similar findings are shown. Hoechst 33258 was used for counterstaining. mIgG1: mouse IgG1. NRS: normal rabbit serum. NGS: normal goat serum. Bar: $10 \mu \mathrm{m}$.

Fig. 2. Detection of HTLV-1 Gag protein on the surface of SGECs. A: After the SGECs were co-cultured for $1 \mathrm{~h}$ with HCT-5 cells, the fixed cells were stained with mouse antibody against HTLV-1 Gag proteins (mGag) and rabbit 
antibody against an SGEC marker, cytokeratin 8/18 (rcyto8/18). Arrowhead: Emitted HTLV-1 Gag particles from HCT-5. Insets: Magnified images of HTLV-1 Gag particles. B: Representative images of the interface between HCT-5 cells and SGECs. Arrowheads: HTLV-1 Gag particles transmitted toward SGECs. Insets: Magnified views of HTLV-1 particles emitted toward SGECs. C: Images of the co-culture of MOLT-4 cells and SGECs are shown as a control (upper panel). Only MOLT-4 cells were stained with mouse anti-HTLV-1 Gag antibody (lower panel). Bright field images were simultaneously captured. Representative results of five independent experiments with similar findings are shown. Hoechst 33258 was used for counterstaining. Bar: $10 \mu \mathrm{m}$.

Fig. 3. Co-expression of extracellular matrix and linker proteins after co-culture. A: After the SGECs were co-cultured for $1 \mathrm{~h}$ with HCT-5 cells, the fixed cells were stained with mouse anti-HTLV-1 Gag antibody (mGag), rabbit anti-galectin-3 (rgalectin-3), agrin antibodies (ragrin) and goat anti-tetherin antibody (gtetherin) followed by FITC-conjugated anti-mouse and TRITC-conjugated anti-rabbit or goat secondary antibodies. Arrowhead: HTLV-1 Gag protein, matrix, and linker proteins or a merged view of these 
proteins. B: SGECs were also co-cultured with MOLT-4 cells for $1 \mathrm{~h}$. The same primary and secondary antibodies were used as for the HCT-5 cells. Representative results of five independent experiments with similar findings are shown. Hoechst 33258 was used for counterstaining. mIgG1: mouse IgG1. NRS: normal rabbit serum. NGS: normal goat serum. Bar: $10 \mu \mathrm{m}$.

Fig. 4. Detection of virological synapse formation. A: After the SGECs were co-cultured for $1 \mathrm{~h}$ with HCT-5 cells, the fixed cells were stained with mouse anti-HTLV-1 Gag (mGag) or ICAM-1 antibody (mICAM-1), or with rabbit anti-cyto8/18 (rcyto8/18), Talin (rtalin), LFA-1 (rLFA-1) or alpha-tubulin (rtubulin) antibodies followed by FITC-conjugated anti-mouse and TRITC-conjugated anti-rabbit secondary antibodies. Arrowhead: The interface between HTLV-1 Gag protein or a merged view of the surface HCT-5 cells and SGECs. B: The same primary and secondary antibodies were used as described for panel A. Representative results of four independent experiments with similar findings are shown. Hoechst 33258 was used for counterstaining. Bar: $10 \mu \mathrm{m}$.

Fig. 5. Detection of HTLV-1 virions by scanning electron microscopy. A: 
HCT-5 cells and (B) MOLT-4 cells viewed by optical microscopy. Insets: Magnified view of respective cells. C: Typical view of an HCT-5 cell by SEM. D: Magnified view of the surface of an HCT-5 cell. Arrowheads: 100-nm particles. E, F: Immune electron microscopy images were shown. HCT-5 cell pellet fixed with 4\% PFA was used for post embedding staining without mouse anti-HTLV-1 Gag antibody (E) or with anti-HTLV-1 Gag antibody (F) followed by $10 \mathrm{~nm}$ gold particle conjugated anti-mouse secondary antibodies. Left panels and right panels showed SEI and backscatterd electron image (BEI) of same location, respectively. Arrowheads showed positive spots in BEI and outlined arrowheads showed corresponding points in SEI. EG,FH: Proximity between HCT-5 and SGECs. Secondary electron images (SEIs) are shown. Alternatively, a lower secondary electron image (LEI) was used when the SEI showed the charge-up phenomenon that expressed an unstable abnormal contrast by a deviated track of a secondary electron. GI,HJ: Detection of HTLV-1 virions at the interface between HCT-5 and SGECs by SEM. White squares show images taken under increasingly greater magnification. Arrowheads indicate the enlarged view of virions. Representative results of three independent experiments with similar findings are shown. 
Fig. 6. Detection of HTLV-1 virions by transmission electron microscopy. A:

The typical appearance of an HCT-5 cell with virions on the surface by transmission electron microscopy (TEM) is shown in the left panel. A magnified view of the surface of another HCT-5 cell is show in the right panel. The inset shows an enlarged view of the virions. B: Differences in size were observed in mature HTLV-1 virions with an envelope and rod-shaped nucleus on the foot processes of HCT-5. Left inset: An enlarged view of HTLV-1 virions. Right inset: Hemispherical virions (arrowheads) were budding from the surface of the HCT-5 cells. C and D: Immune electron microscopy was shown. HCT-5 cell pellet fixed with 4\% PFA was used for post embedding staining without mouse anti-HTLV-1 Gag antibody (C) or with anti-HTLV-1 Gag antibody (D) followed by 10nm gold particle conjugated anti-mouse secondary antibody. $\mathbf{E E : ~ C o n t a c t ~ b e t w e e n ~ a n ~}$ HCT-5 cell and an SGEC is shown. PF: Detection of HTLV-1 virions at the interface between HCT-5 cells and SGECs. EG: Black arrowheads: HTLV-1 virions of approximately 100-nm diameter on the surface of an HCT-5. FH: Virions on the SGECs side. The outlined arrowheads in $\mathbf{A}, \mathbf{P F}$ and $\mathbf{F H}$ indicate the thin film-like structure. Representative results of three independent 
experiments with similar findings are shown.

Suppl. Fig. S1. Expression of biofilm and VS-related molecules before and after co-culture. A: The expression of HTLV-1 Gag was first examined without co-culture with HCT-5 or MOLT-4 cells. After fixation, the SGECs were reacted with mouse anti-HTLV-1 Gag antibody (mGag) or mouse IgG1 followed by FITC-conjugated anti-mouse secondary antibodies. The expression of biofilm and VS-related molecules including galectin-3, agrin and tetherin, LFA-1, talin and alpha-tubulin were then examined by reacting with primary antibodies including rabbit anti-galectin-3 (rgalectin-3), agrin (ragrin), LFA-1 (rLFA-1), Talin (rtalin) and alpha-tubulin (rtubulin) antibodies, goat anti-tetherin antibody (gtetherin), normal rabbit serum (NRS) or normal goat serum (NGS) followed by TRITC-conjugated anti-rabbit or goat secondary antibodies. Representative results of three independent experiments with similar findings are shown. B: HTLV-1 Gag protein and galectin-3, agrin, and tetherin co-expressed cases that are not shown in Figure 3. In the six cases, white arrowheads and outlined arrowheads indicate merged and unmerged cases, respectively.

Hoechst 33258 was used for counterstaining. Bar: $10 \mu \mathrm{m}$. 


\section{Suppl. Fig. S2. Inhibition of tetherin on SGECs by neutralizing antibody.}

The SGECs were co-cultured for 1 and $24 \mathrm{~h}$ with HCT-5 cells in the presence or absence of indicated concentration of mouse anti-tetherin neutralizing antibody. The fixed cells were stained with mouse anti-HTLV-1 Gag (mGag: green) or with goat anti-tetherin antibody (gtetherin: red) followed by FITC-conjugated anti-mouse and TRITC-conjugated anti- goat secondary antibodies. Arrowheads showed co-expression of HTLV-1 Gag and tetherin. Representative results of two independent experiments with similar findings are shown. Hoechst 33258 was used for counterstaining. Bar: $10 \mu \mathrm{m}$.

Suppl. Fig. S3. Co-culture of SGECs with MOLT-4 cells, observed by EM. A-D: Magnified view between a MOLT-4 cell and an SGEC by SEM. The SEI image was taken after the specimen was sputtered with platinum. A: Typical view of a MOLT-4 cell. B: SGECs co-cultured with MOLT-4 cells. Inset: Enlarged view of the surface of an SGEC. C,D: Contact of MOLT-4 cell with an SGEC. Asterisks: Interface. E-H: Magnified view between a MOL-4 cell and 
SGEC obtained by TEM after making thin-sliced sections from polymerized epoxy resin. E: TEM image of a MOLT-4 cell. F: SGECs co-cultured with MOLT-4 cells. G: Interface between a MOLT-4 cell and an SGEC. H: Enlarged view of panel G. Representative results of two independent experiments with similar findings are shown.

\section{Suppl. Fig. S4. Formation of cellular conduits between HCT-5 and SGECs.}

After the SGECs were co-cultured for $0,3,20$ and 60 minutes with HCT-5 cells, the fixed cells were stained with mouse anti-HTLV-1 Gag (mGag) or with rabbit anti-cyto8/18 (rcyto8/18), LFA-1 (rLFA-1) antibodies followed by FITC-conjugated anti-mouse and TRITC-conjugated anti-rabbit secondary antibodies. Enlarged view of cellular conduits was shown in inset at 60 minutes. Bright field images were simultaneously captured. Representative results of three independent experiments with similar findings are shown. Hoechst 33258 was used for counterstaining. Bar: $10 \mu \mathrm{m}$. 
A

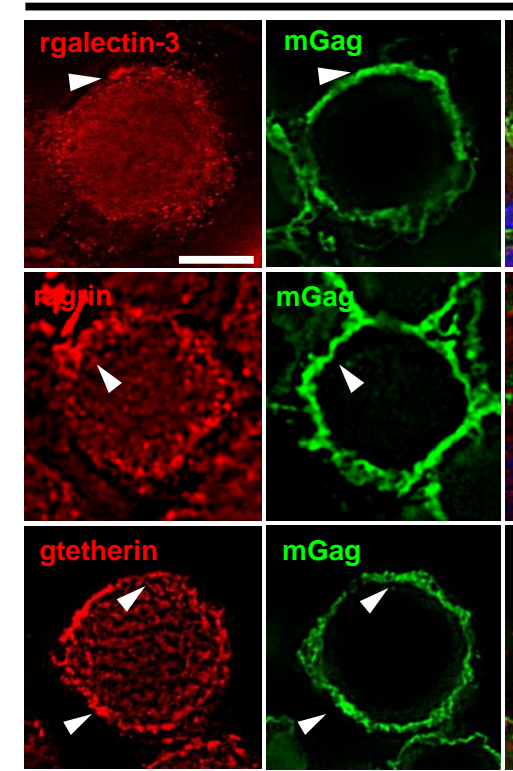

NRS mlgG
HCT-5

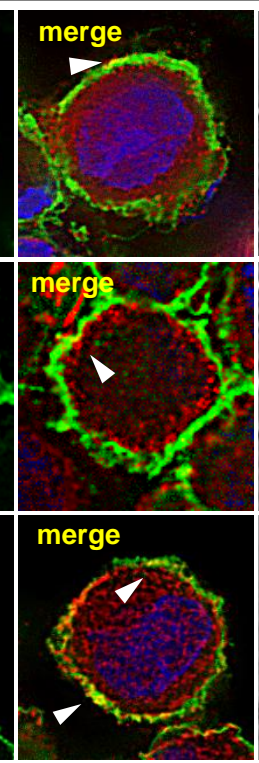

Bright field + IF
B

ב

Bright field + IF

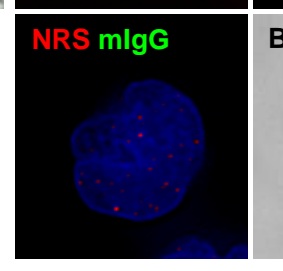

MOLT-4

\begin{tabular}{|l|l|l|}
\hline merge & Bright field & Bright field + IF \\
\hline merge & Bright field & Bright field + IF \\
\hline & & \\
\hline merge & Bright field & Bright field + IF \\
\hline & \\
\hline
\end{tabular}

Bright field + IF NGS mlgG

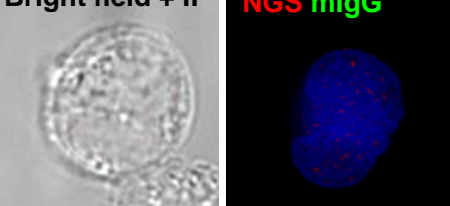

Bright field + IF

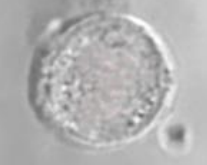



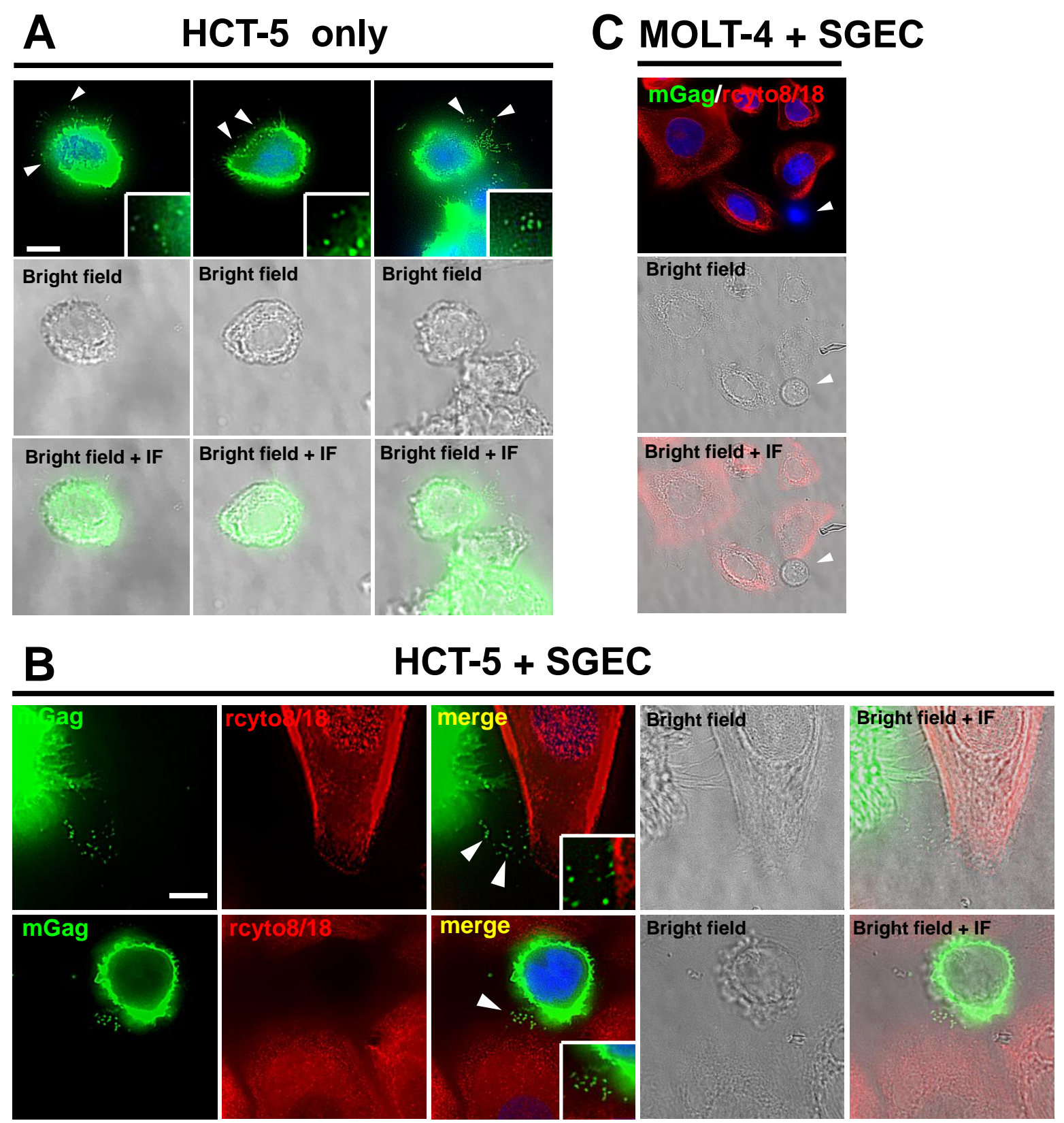


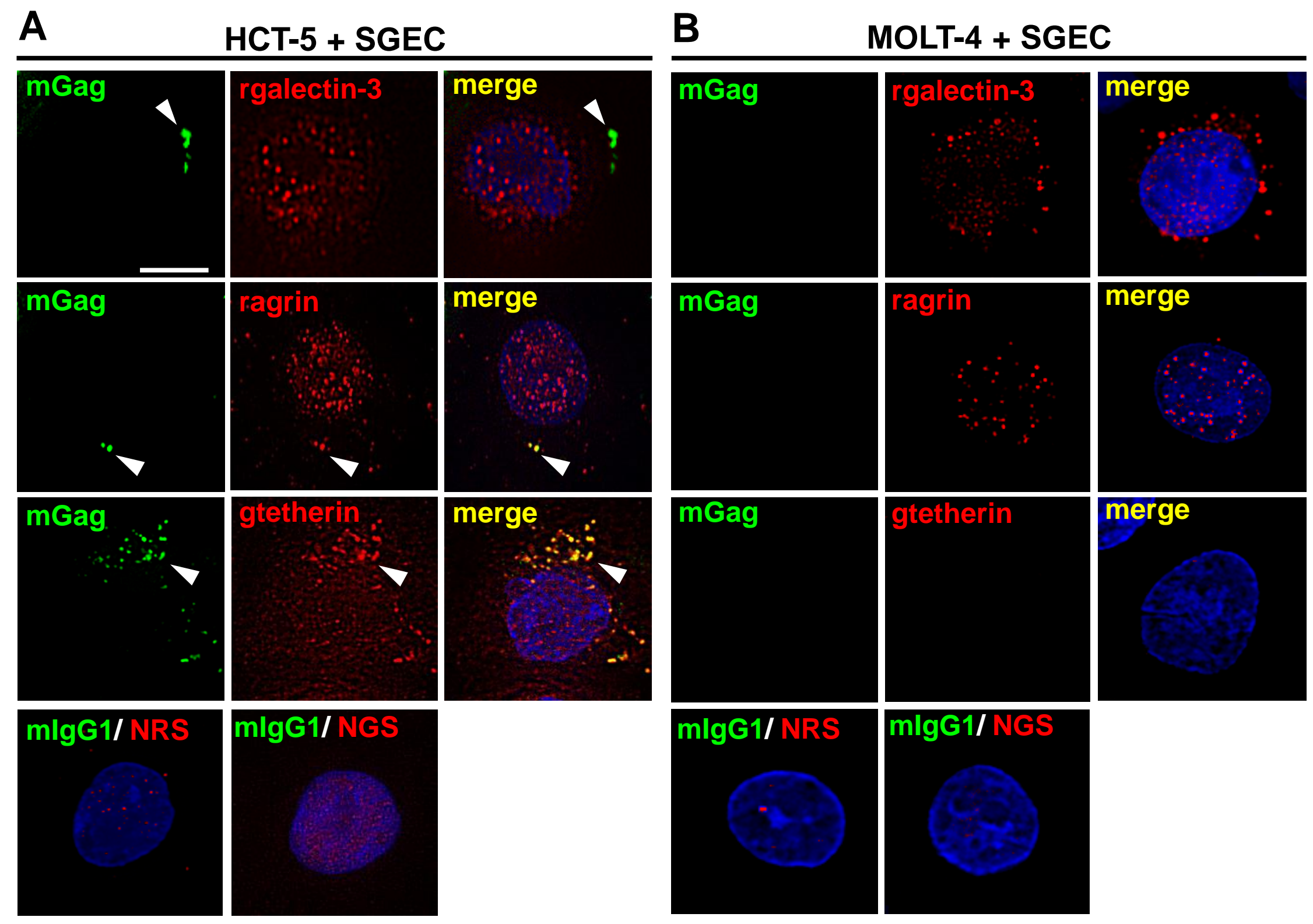




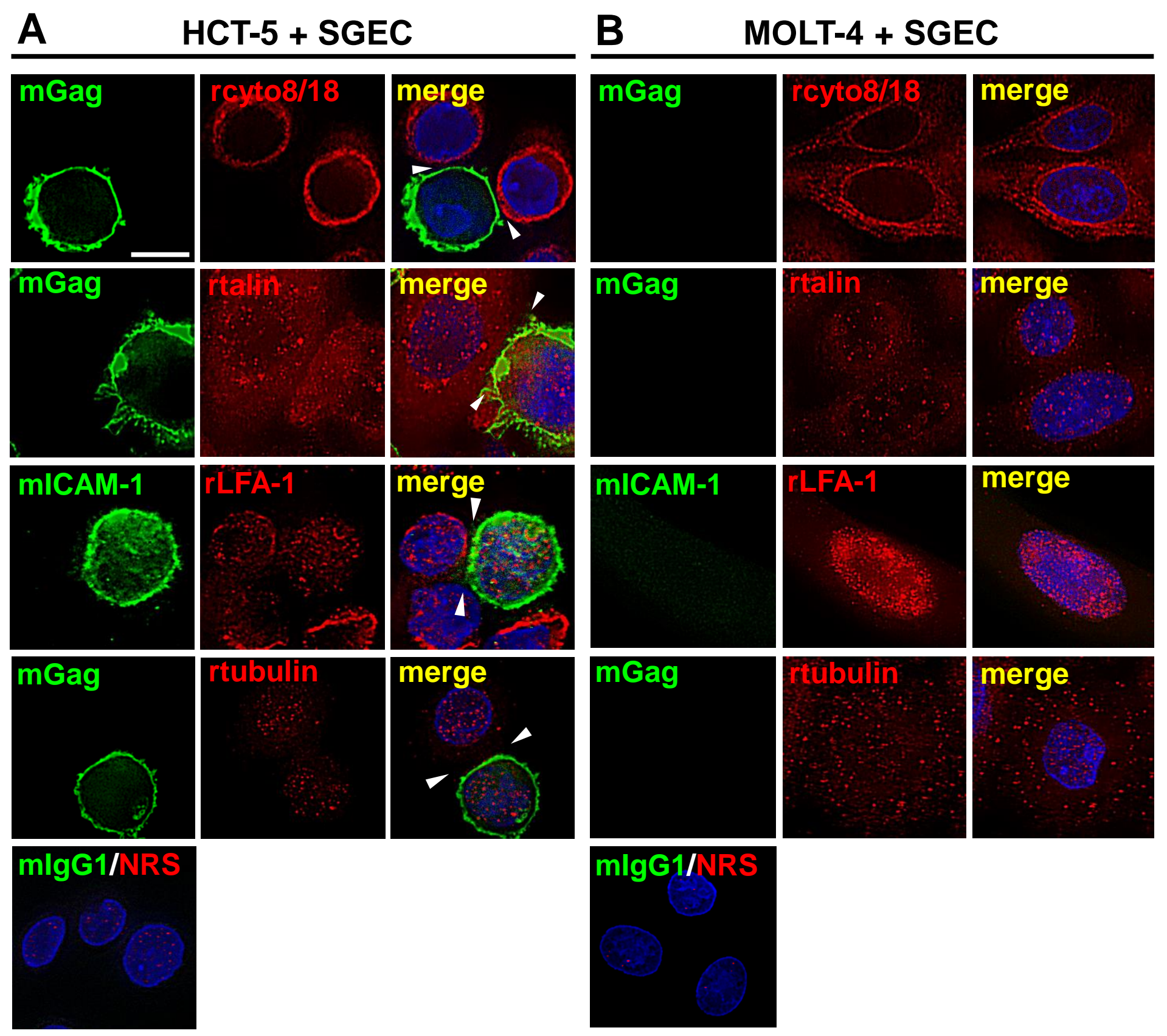


Fig 5
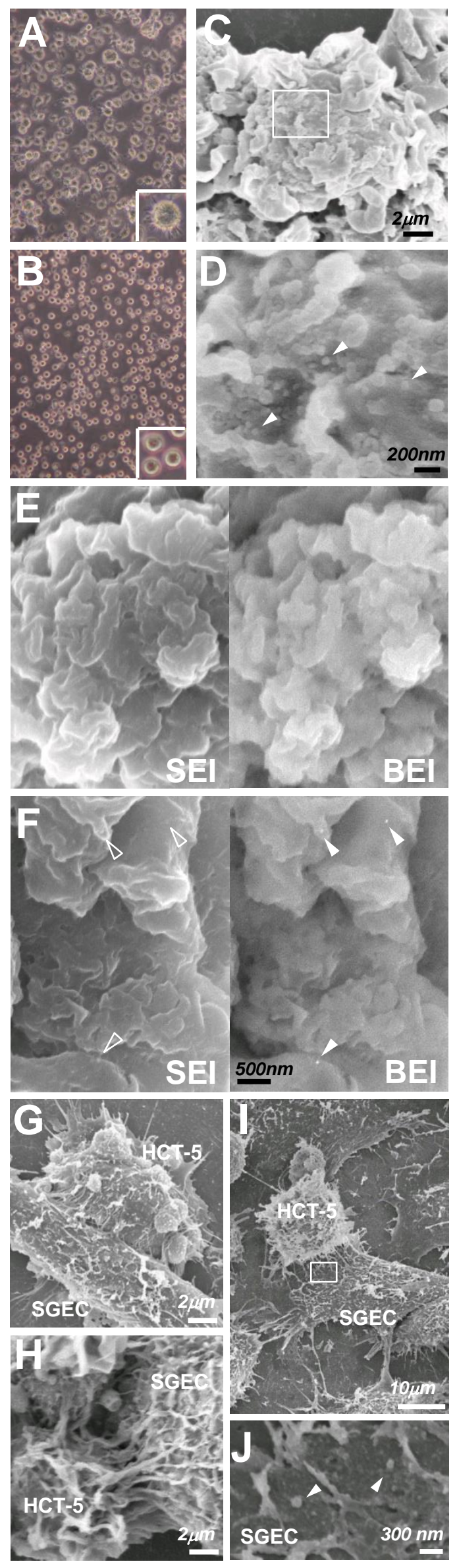
Fig 6

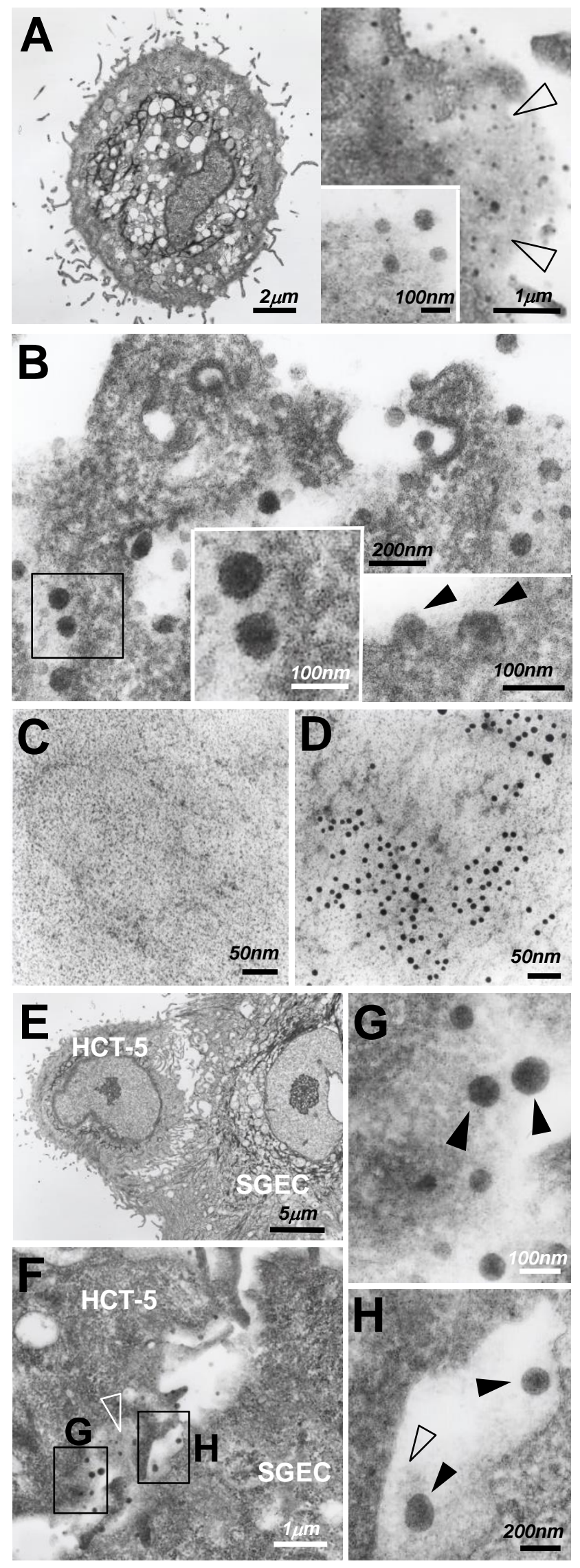




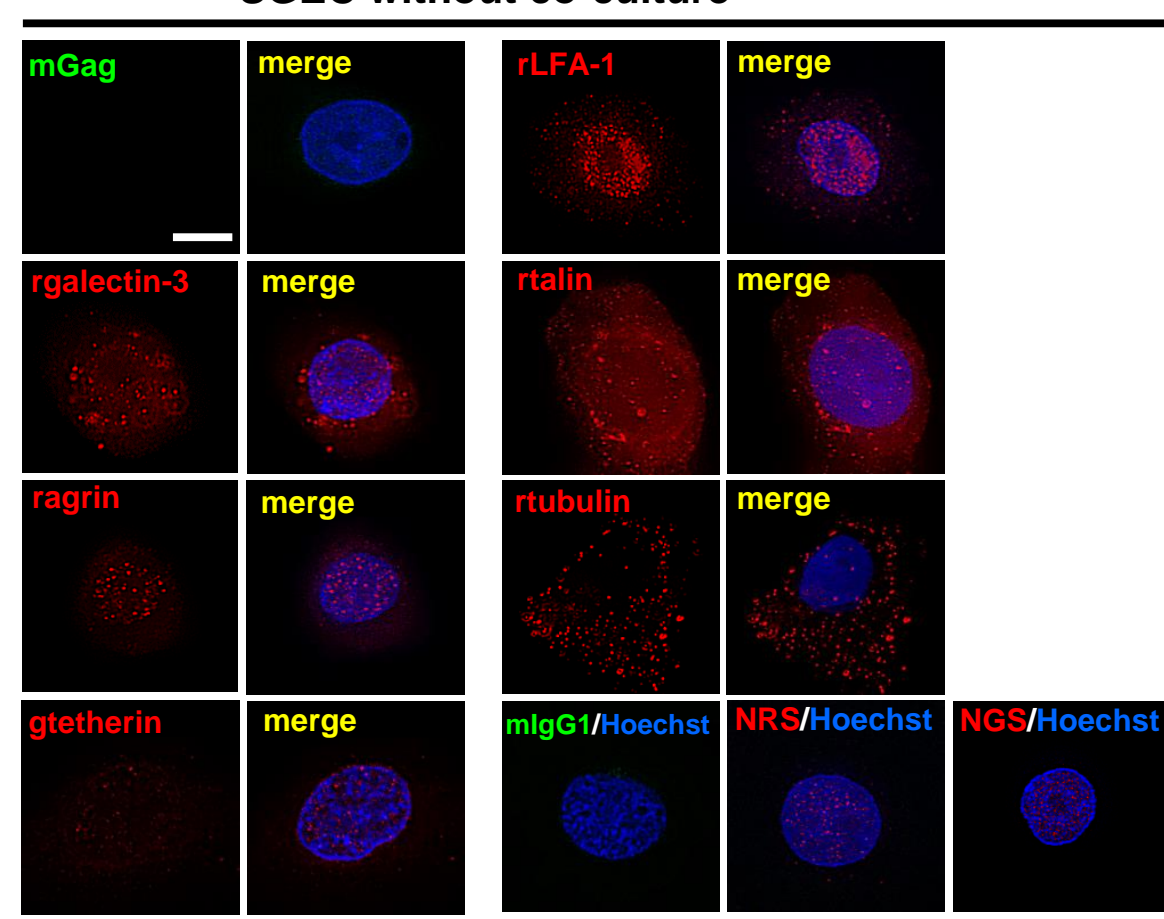

B

HCT-5 + SGEC

mGag

rgalectin-3

mGag

ragrin

mGag

gtetherin

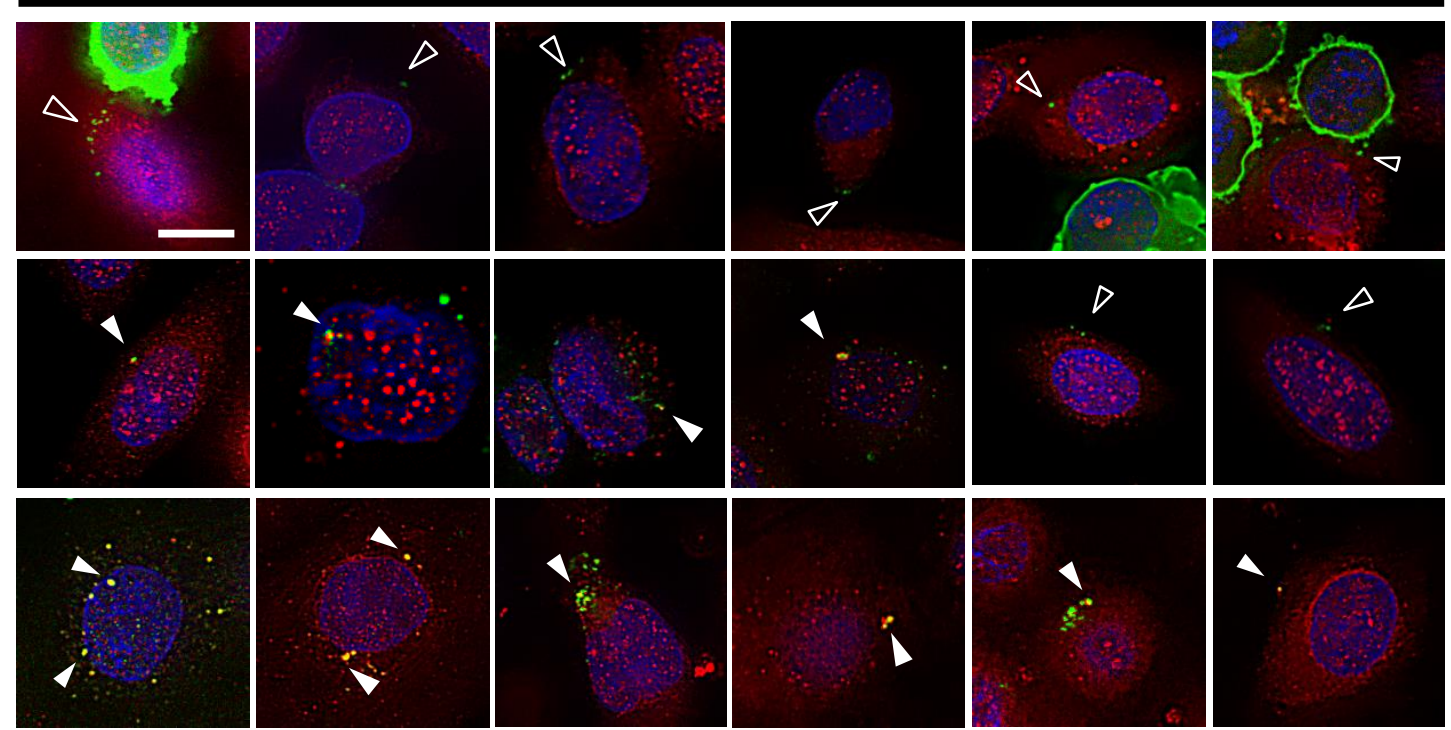


HCT-5 (+)

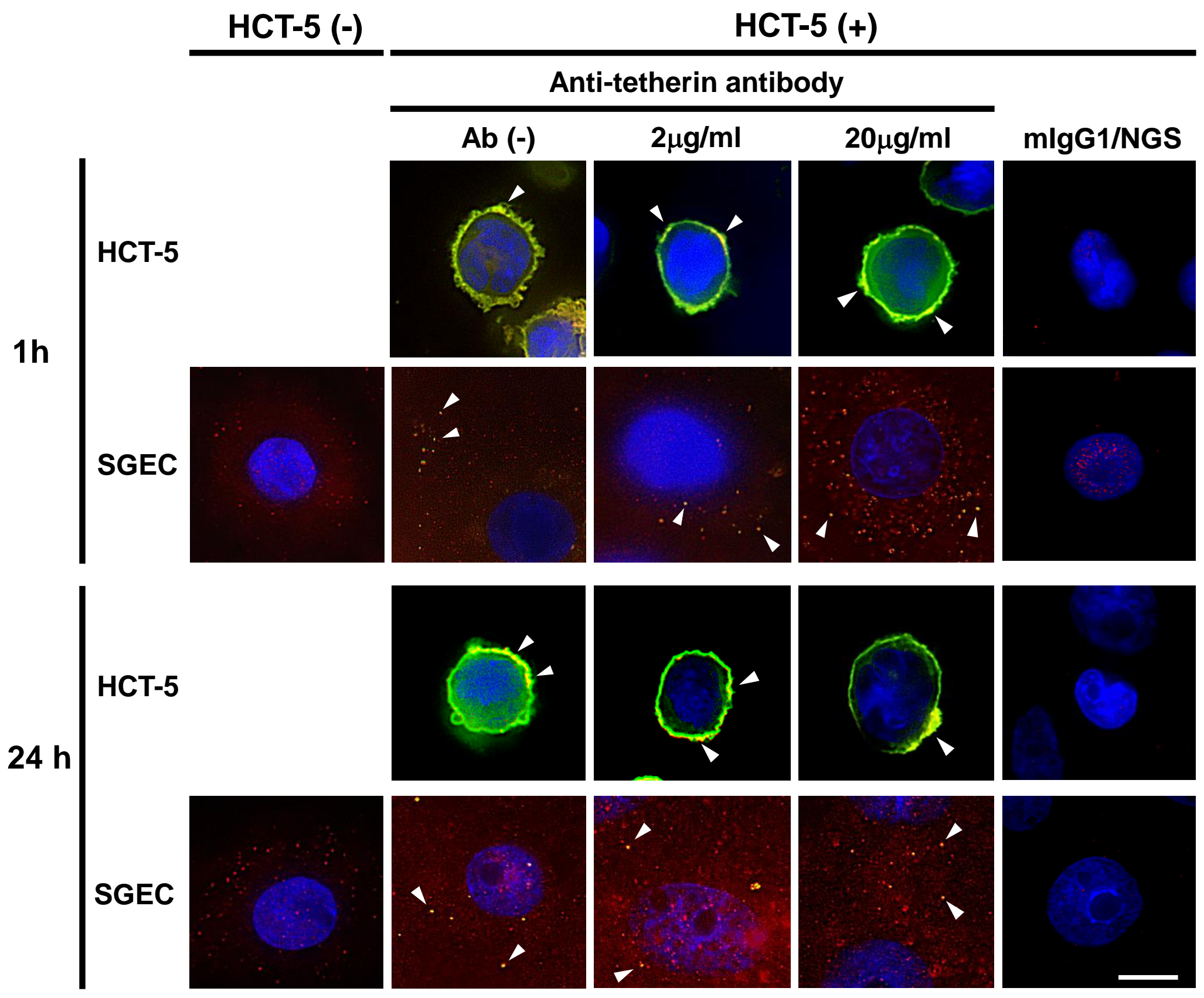



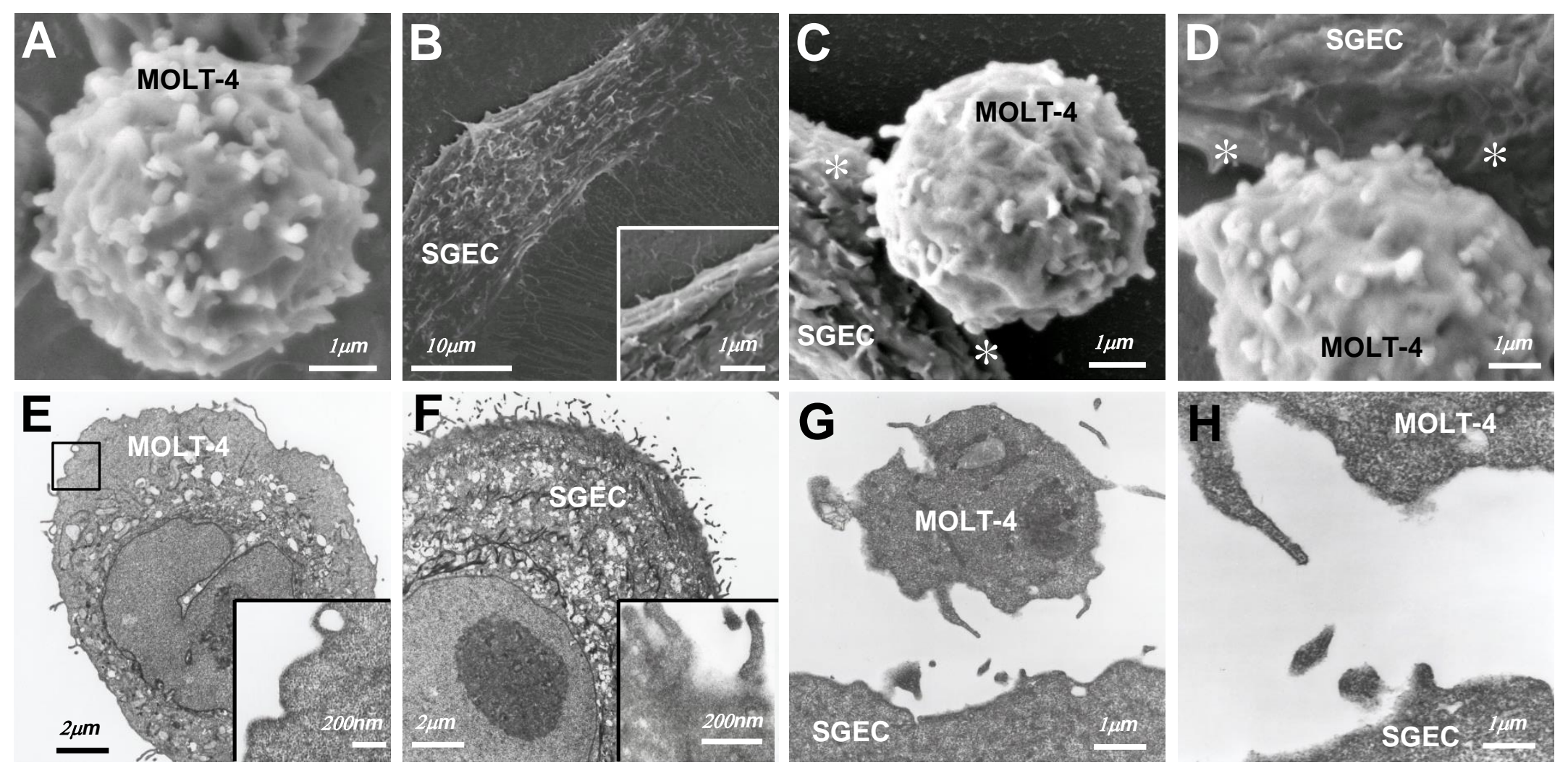


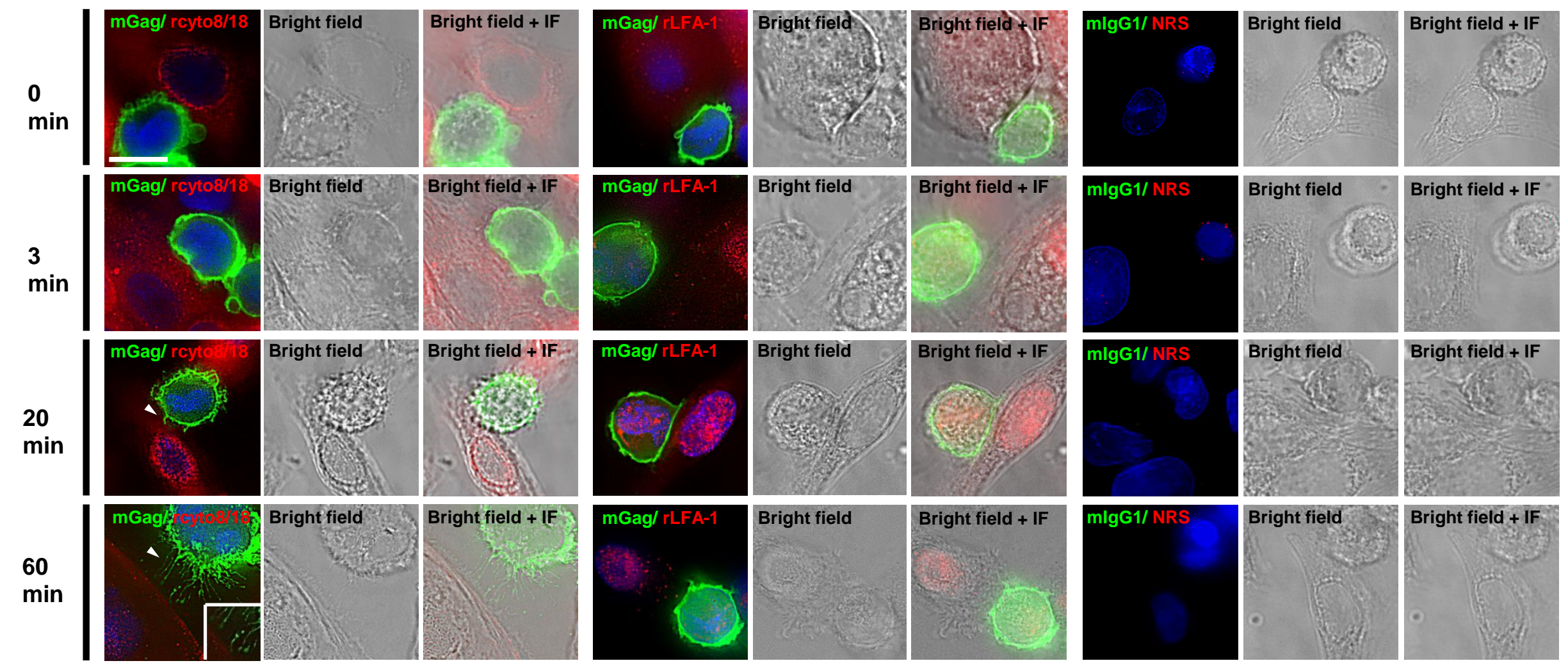

